\title{
Dakhlia Jamil (dir.) : À la recherche des publics populaires (1). Faire peuple
}

\section{Amandine Kervella}

\section{(2) OpenEdition}

\section{Journals}

Édition électronique

URL : http://journals.openedition.org/edc/6607

DOI : $10.4000 /$ edc.6607

ISSN : 2101-0366

Éditeur

Université de Lille

Édition imprimée

Date de publication : 1 décembre 2016

Pagination : 185-190

ISBN : 978-2-917562-16-1

ISSN : $1270-6841$

Référence électronique

Amandine Kervella, «Dakhlia Jamil (dir.) : À la recherche des publics populaires (1). Faire peuple », Études de communication [En ligne], 47 | 2016, mis en ligne le 01 décembre 2016, consulté le 24 septembre 2020. URL : http://journals.openedition.org/edc/6607 ; DOI : https://doi.org/10.4000/edc.6607

Ce document a été généré automatiquement le 24 septembre 2020.

(c) Tous droits réservés 


\title{
Dakhlia Jamil (dir.) : À la recherche des publics populaires (1). Faire peuple
}

\author{
Amandine Kervella
}

\section{RÉFÉRENCE}

Dakhlia J. (dir.) (2015). À la recherche des publics populaires (1). Faire peuple, Nancy, PUN Éditions universitaires de Lorraine.

1 Cet ouvrage, divisé en trois parties et composé de treize contributions, constitue le premier tome des actes du colloque international A la recherche des publics populaires/ Looking for Popular Publics organisé par le Centre de recherche sur les médiations (CREM), en 2011 et 2012. Comme Jamil Dakhlia (professeur en sciences de l'information et de la communication à l'Université Sorbonne Nouvelle - Paris 3), qui dirige ce premier volume, l'explique en introduction, l'intérêt de ce colloque et, donc des ouvrages qui en découlent, se situe dans l'ambition affichée de croiser deux notions équivoques, polysémiques et largement débattues, celle de «public» et de « populaire ». Il s'agit plus précisément de « vérifier si la catégorie 'public populaire' est toujours pertinente, voire heuristique, pour nourrir la recherche en sciences sociales » (p. 9), en mobilisant pour ce faire les ressources de l'interdisciplinarité. Dans ce premier opus, l'enjeu est spécifiquement de «cerner la dimension stratégique de la constitution de publics populaires » (p. 10).

2 Les contributions retenues pour la publication ont été classées en fonction des types de convocation des publics populaires qu'elles mobilisent: théories, stratégies et dispositifs du «faire peuple».

3 La première partie de l'ouvrage s'intéresse à la manière dont peuvent être théorisés les publics populaires et les «appels au peuple». Roger Bautier ouvre la réflexion en décrivant comment la question de la participation des publics populaires au fonctionnement de l'espace public a été traitée depuis les débats politiques de la France dudébut du XIX ${ }^{\mathrm{e}}$ siècle, jusqu'aux travaux des sociologues de la culture de la fin du XX 
siècle, en passant par ceux d'Antonio Gramsci. Son analyse rappelle la place centrale occupée à ce niveau par la question du langage du peuple et de celui qui lui est destiné. Il pose ainsi la délicate question du rapport aux normes de la communication. Jean Caune s'intéresse ensuite à la notion de public populaire au théatre, en tant que « fait social total», au sens proposé par Marcel Mauss ${ }^{1}$, dans une triple perspective historique, politique et esthétique. Pour circonscrire la réalité de ce public, Jean Caune rappelle les projets portés pour lui par trois grands metteurs en scène et responsables d'institutions théâtrales, Firmin Gémier, Jacques Copeau et Jean Vilar. S'appuyant sur ces exemples, il propose l'hypothèse selon laquelle « le théâtre populaire relève moins d'une esthétique que d'une éthique » (p. 38) et invite à poser la question de la " place de la pratique théâtrale dans la nécessité de reconnaissance des droits culturels de tous et de chacun »(p. 39). Adela Rogojinaru complète l'analyse des théories du public en proposant de saisir le caractère populaire des publics numériques contemporains, à partir d'approches théoriques interdisciplinaires. La contribution de Svetlana Dimitrova clôt la première partie de l'ouvrage par une réflexion sur la notion de "populisme ", menée à partir du cas empirique bulgare postsocialiste. Pour l'auteure de ce chapitre, il s'agit avant tout de questionner les actions, les discours et les acteurs qui contribuent à placer des politisations contestataires sous ce label. C'est donc bien au travail d'étiquetage associé au "populisme » que Sveltlana Dimitrova s'intéresse, tout en invitant à déplacer le centre de l'attention de la catégorie vers ceux qui l'emploient, dans la lignée des travaux d'Annie Collovald ${ }^{2}$.

La seconde partie de l'ouvrage s'intéresse plus particulièrement aux stratégies de mobilisation des publics populaires. Elle interroge différentes opérations d'« appel au peuple ", pour en mettre au jour la cohérence et les différentes logiques. Isabelle Mathieu l'inaugure à travers une contribution qui fait directement écho à celle de Jean Caune. Interrogeant le théâtre populaire dans une perspective diachronique, elle confronte les sources du théâtre populaire - principalement à travers l'analyse de deux visions différentes de ce théâtre, portées par Maurice Pottecher et Romain Rolland - à la notion contemporaine de médiation culturelle et à la fonction qui lui est attribuée dans le cadre des politiques publiques de la culture. Cette démarche lui permet de formuler l'hypothèse conclusive de l'importance de la prise en compte de la dimension territoriale de la médiation culturelle lorsqu'il s'agit de susciter la réunion du peuple autour d'une culture partagée. Les deux contributions suivantes, celle de Frédérique Giraud et de Christina Holtz-Bacha et Eva-Maria Lessinger proposent d'envisager la question de l'«appel au peuple» à travers l'analyse de la stratégie d'individus singuliers. Frédérique Giraud revient sur la manière dont Émile Zola était à la « recherche du peuple», en écrivant des romans à la fois «sur» et " pour » le peuple. Par-delà une stratégie de captation du public populaire à des fins mercantiles, Frédérique Giraud montre comment ce parti pris relève aussi d'une stratégie de positionnement dans le champ littéraire de son époque et d'une contestation de l'élitisme intellectuel et social. Christina Holtz-Bacha et Eva-Maria Lessinger interrogent quant à elles la stratégie de "peopolisation » déployée par le baron KarlThéodor zu Guttenberg, alors ministre de l'Économie, à la fin des années 2000, en Allemagne, pour en souligner les conséquences sur une carrière politique individuelle et sur le système politique dans son ensemble. Il s'agit aussi pour les auteures de souligner le poids du fonctionnement des médias et des horizons d'attentes culturellement marqués dans ce processus. Delphine Saurier propose ensuite une contribution analysant l'émergence d'un public spécifique : celui des personnes placées 
sous main de justice et bénéficiant de l'accès à des projets patrimoniaux. Il ne s'agit pas pour Delphine Saurier d'analyser la matérialité de ce public populaire, mais de montrer comment il se construit, pour un temps déterminé, à travers les paroles et les actes des professionnels des ministères de la Culture et de la Justice qui déploient les projets qui cherchent à le constituer. Or, ce qui se donne ainsi à voir est bien le «mirage $»^{3}$ que constitue un public éphémère dont la constitution même est stigmatisante pour ceux qui le composent. Romain Badouard clôture la seconde partie de l'ouvrage en explorant les modalités de constitution des publics en ligne, à travers le cas d'étude d'une plateforme participative mise en ligne par la Commission européenne à destination des internautes, citoyens "ordinaires", de divers États membres, au milieu des années 2000. Il montre la difficulté de «fabriquer » ce public, tant au regard des stratégies de communication déployées pour ce faire, des caractéristiques de l'outil mobilisé, que des pratiques réellement déployées par les internautes. Il remarque en outre l'importance de la controverse dans le processus d'émergence de publics en ligne.

sous l'angle des dispositifs. Sébastien Rouquette puis Christine Servais interrogent le poids des dispositifs dans certaines interpellations médiatiques des publics populaires. Constatant la faiblesse de la représentation des citoyens populaires dans les débats télévisés français de la seconde moitié du XXe siècle, mais également sur l'internet, Sébastien Rouquette souligne le poids de facteurs explicatifs médiatiques et sociaux, entre critères de sélection des « bons invités » par les journalistes et réflexes d'autocensure des internautes issus des catégories populaires. S'intéressant à l'appel au peuple proposé par les médias de masse, Christine Servais développe une approche réflexive invitant à analyser la réception à partir des notions $\mathrm{d}^{\prime}$ ' adresse » (Derrida $\left.{ }^{4}\right)$ et de "scène du sensible " (Rancière $\left.{ }^{5}\right)$. Les deux dernières contributions se fondent sur l'étude empirique de deux dispositifs pensés à destination des publics populaires. Céline Schall et Jean-Christophe Vilatte analysent le cas d'une exposition temporaire d'archéologie à travers le discours de ses concepteurs et une étude sémiotique pour confronter la notion de "grand public» à celle de "public populaire » et en faire ressortir la dimension plus morale qu'opérationnelle. Gaëlle Crenn explore la production et la réception de l'exposition «Abbaworld ", présentée en 2010-2011 au muséePowerhouse de Sydney, mettant en lumière de nouvelles manières d'exposer la culture populaire au musée et de nouvelles figures du public. À travers cette étude de cas, c'est bien aussi le rôle du musée dans la société qui est questionné.

6 Cet ouvrage apporte une contribution très utile à l'ensemble des travaux pouvant être menés autour de la notion complexe de "public populaire ». La partition de l'ouvrage en trois parties regroupant les contributions non pas en fonction de leurs objets, mais de trois principaux types de convocation des publics populaires - théories, stratégies et dispositifs du "faire peuple »- se révèle très stimulante. Le choix d'une perspective interdisciplinaire et la variété des études de cas mobilisées sont également féconds. Cet ouvrage intéressera donc des chercheurs de différents champs disciplinaires : sciences de l'information et de la communication, sociologie ou encore sciences politiques. 


\section{NOTES}

1. Mauss M. (2007 [1925]). Essai sur le don. Forme et raison de l'échange dans les sociétés archaïques, Paris, PUF.

2. Collovald A. (2004). Le "populisme du FN» un dangereux contresens, Bellecombe-en-Bauges, Éditions du Croquant.

3. Fabiani J.-L. (2008). L'Éducation populaire et le théâtre. Le public d'Avignon en action, Grenoble, PUG.

4. Voir par exemple : Derrida J. (1967). De la grammatologie, Paris, Éditions de Minuit.

5. Voir par exemple : Rancière J. (2000). Le partage du sensible. Esthétique et politique, Paris, Éditions La Fabrique.

\section{AUTEURS}

\section{AMANDINE KERVELLA}

Univ. Lille, EA 4073 - GERiiCO - Groupement d'Études et de Recherche Interdisciplinaire en Information et Communication, F-59000 Lille, France amandine.kervella@univ-lille1.fr 